\title{
CARACTERIZACIÓN DEL FLUIDO DE TRABAJO PARA MOTORES DE CICLO OTTO A CUATRO TIEMPOS Y SU UTILIZACIÓN EN EL CICLO INDICADO PREVISTO.
}

Diego C. Caputo ${ }^{a}$, Rodolfo O. Berberi ${ }^{a}$, Nestor A. E. Ferréb, Alberto Puricellib $^{\mathrm{b}}$ Verónica Fonteriz ${ }^{\mathrm{b}}$, Romina Ferreyra ${ }^{\mathrm{b}}$, Rodolfo N. Aguirre ${ }^{\mathrm{b}}$, Blas E. $\mathrm{Calvo}^{\mathrm{b}}$, José Mauro Bruno

\section{RESUMEN}

El presente trabajo se encuentra inserto en el proyecto de investigación denominado "Modelo físico matemático del ciclo previsto para motores de combustión interna" (CIPREV), realizado en conjunto entre el Departamento de Ingeniería Mecánica de la Universidad Tecnológica Nacional FRBA y la Facultad de Ingeniería de la Universidad de la Marina Mercante. Como ya es sabido el motor alternativo basa su principio de funcionamiento en el aprovechamiento mecánico de la expansión de los gases de combustión dentro del cilindro para transmitir movimiento a los órganos del sistema biela manivela. El presente estudio se propone indagar en la naturaleza y características del fluido de trabajo de la máquina para que sus resultados puedan ser utilizados como datos para el cálculo del ciclo indicado de los motores. El procedimiento experimental se ha basado en la determinación del coeficiente politrópico del proceso de compresión y la determinación de la composición de los gases de combustión durante un ensayo de potencia al freno con obtención del consumo específico.

Palabras Clave: motor, ciclo indicado, exponente politrópico, potencia, compresión.

\section{INTRODUCCIÓN}

El conocimiento y caracterización del fluido de trabajo resultan de vital importancia en el desarrollo de los modelos de cálculo del ciclo indicado en motores de combustión interna. Desde los modelos más teóricos de la máquina ideal en cuyo ciclo evoluciona como fluido de trabajo el aire, hasta los modelos más reales como el ciclo límite o el ciclo indicado previsto [1], definir el fluido de trabajo y sus características resulta imprescindible al momento de determinar el calor producido, el trabajo, la potencia, el consumo y otras características inherentes al ciclo y la máquina.

\footnotetext{
${ }^{a}$ Universidad de la Marina Mercante, Facultad de Ingeniería, Av. Rivadavia 2258 Ciudad Autónoma de Buenos Aires, Argentina.dcaputo@udemm.edu.ar, rodolfo.berberi@gmail.com

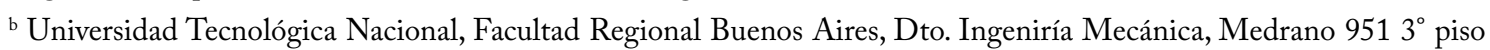
of. 303 C.A.B.A, Argentina. nesferre@gmail.com, copimagen5@gmail.com, vero_fonteriz15@hotmail.com, roelizabeth_15@hotmail.com, aguirre.rodo@gmail.com, blascalvo88@hotmail.com,maurojbruno@yahoo.com
} 
Como es suficientemente conocido el ciclo de trabajo de un motor de combustión interna que opera según el ciclo Otto de cuatro tiempos, está constituido por dos transformaciones a volumen constante y dos politrópicas, una de compresión y otra de expansión.

Su trabajo está determinado por la cantidad de fluido que opera y por las temperaturas extremas que alcanza. El mismo puede ser representado en un diagrama presión - volumen, y su área determina el trabajo y la potencia que se puede obtener.

El presente estudio se apoya en el trabajo experimental desarrollado en el laboratorio de Termofluídos y Máquinas Térmicas dependiente de la Facultad de Ingeniería de la Universidad de la Marina Mercante y centra su atención en aportar datos para una mayor comprensión acerca de la naturaleza del agente de trabajo real del motor. Aquí se muestran los resultados obtenidos en la primera etapa de determinación del coeficiente politrópico, centrando el estudio en la carrera de compresión.

\section{PLANTEO DEL PROBLEMA Y MODELOS PROPUESTOS}

\subsection{Caracterización del proceso de com- presión.}

El proceso de compresión corresponde al segundo tiempo del ciclo operativo de cuatro tiempos. Tiene lugar al finalizar la carrera descendente de admisión, cuando el pistón llega al punto muerto inferior $\mathrm{PMI}$, y comienza su carrera ascendente. El cambio de dirección va reduciendo el volumen total del cilindro $V_{1}$, e interactúa con la carga nueva ingresando al cilindro y con dirección opuesta. Cuando se pro- duce el cierre de las válvulas de admisión comienza un incremento sostenido de la presión hasta alcanzar la máxima presión de compresión $\mathrm{P}_{2}$ cuando el pistón llega nuevamente al punto muerto superior PMS, y el volumen total del cilindro $V_{1}$ queda reducido al de la cámara de combustión $V_{2}$.

En el proceso de compresión participa una masa determinada de fluido de trabajo, e influyen en forma determinante la velocidad con que se realiza, los parámetros iniciales de presión $\mathrm{P}_{1} \mathrm{y}$ temperatura $\mathrm{T}_{1}, \mathrm{y}$ la permeabilidad térmica del sistema. Todas estas condiciones de borde determinan la máxima presión $\mathrm{P}_{2} \mathrm{y}$ temperatura $\mathrm{T}_{2}$ que pueden verificarse en el interior del cilindro. La transformación que más se asemeja al proceso de compresión real es del tipo politrópica. Es decir, aquella que se realiza con intercambio de calor con el medio exterior pero manteniendo constantes los calores específicos del fluido de trabajo en el intervalo de temperaturas considerado. $\mathrm{Su}$ formulación genérica es la siguiente:

$$
p \cdot v^{n}=c t e
$$

Y aplicándola a la transformación observada en la carrera de compresión, que sucede entre los volúmenes extremos $V^{1}$ (volumen total o máximo del cilindro) y $V^{2}$ (volumen de la cámara de combustión), puede escribirse:

$$
p_{1} \cdot V_{1}^{n}=p_{2} \cdot V_{2}^{n}
$$

Siendo $n$ el valor del exponente o coeficiente politrópico que caracteriza al fluido de trabajo y al sistema particular. Partiendo de la ecuación (2), podemos obtener la fórmula general para determinar el valor del coeficiente politrópico medio que determina la forma de la curva de compresión y los valores extremos que pueden alcanzarse: 


$$
n=\frac{\log \frac{p_{2}}{p_{1}}}{\log \varepsilon}
$$

La relación de compresión $\varepsilon$, es una relación volumétrica que representa el valor máximo en el que se reduce el volumen total del cilindro hasta alcanzar el volumen de la cámara de combustión, y puede expresarse como sigue

$$
\varepsilon=\frac{V_{1}}{V_{2}}
$$

En su recorrido desde el PMI hasta llegar al PMS, el pistón adopta infinitos puntos intermedios que determinan un sin número de relaciones entre el volumen total del cilindro y el observado en cada punto de su recorrido. En un modo genérico puede escribirse que la presión $p_{y}$ en el interior del cilindro durante la carrera de compresión corresponde a un volumen $V_{x}$, para $V_{2} \leq \mathrm{Vx}$ $\leq V_{1}$, y su valor estará dado por:

$$
p_{y}=p_{1} \cdot\left(\frac{V_{1}}{V_{x}}\right)^{n}
$$

Para esta primera formulación se ha considerado que el exponente politrópico de compresión se mantiene constante durante toda la carrera de compresión.

El trabajo de compresión, suministrado por la máquina al fluido, puede obtenerse a partir de la politrópica de compresión como:

$$
L_{c}(\text { trabajo- compresión })=\int_{V_{2}}^{r_{1}} P y \cdot d v(6)
$$

Sustituyendo $d v$ por $d x$, y $V x$ por $x, \mathrm{y}$ asumiendo constantes los valores de $V_{1}, V_{2}$, y $p_{i}:$

$$
L_{c}=\int_{V_{2}}^{V_{1}}\left(p_{1} \cdot \frac{V_{1}^{n}}{x^{n}}\right) \cdot d x
$$

$$
L_{c}=p_{1} \cdot V_{1}^{n} \cdot \int_{V_{2}}^{V_{1}} x^{-n} \cdot d x
$$

Resolviendo la integral y volviendo a sustituir por los valores correspondientes:

$$
L_{c}=p_{1} V_{1}^{n} \cdot\left(\frac{V_{1}^{-n+1}-V_{2}^{-n+1}}{-n+1}\right)[1]
$$

Asimismo para las transformaciones politrópicas se verifica que el trabajo se pude calcular en función de las temperaturas que corresponden a los estados extremos de la misma y al calor específico a volumen constante del fluido:

$$
L_{c}=c_{v} \cdot\left(T_{2}-T_{1}\right) \cdot m_{t}
$$

Asumiendo para este estudio el modelo de los gases ideales, también debe verificarse para todos los puntos de la politrópica:

$$
R_{g 1}=\frac{p \cdot V}{m_{t} \cdot T}
$$

Donde $m_{t}$ es la masa total de fluido que evoluciona y $R_{g 1}$ la constante particular del sistema.

Por último, conociendo la relación de mezcla $\alpha$ (masa de aire/ masa de combustible) que determina las proporciones en la demanda de aire para una combustión 
completa y el exceso de aire con que ésta se produce, es posible conocer la masa de fluido que evoluciona en el ciclo.

$$
\alpha=\frac{m_{a}}{m_{c}}
$$

Siendo $\lambda$ el exceso de aire con que se produce la combustión, $m_{c}$ la masa de combustible, $m_{a}$ la masa de aire $\mathrm{y} m_{t}$ la masa total de fluido que evoluciona.

$$
\begin{aligned}
& m_{t}=\lambda \cdot m_{a}+m_{c} \\
& m_{t}=m_{c} \cdot(\lambda \cdot \alpha+1)
\end{aligned}
$$

\subsection{Datos de partida e hipótesis simpli-} ficativas.

Se asumen conocidas algunas cuestiones inherentes al cálculo básico de los motores. Para mayor exactitud la relación de compresión $(\varepsilon)$ se ha obtenido midiendo el volumen de cada cámara de combustión y operando como se indica en la tabla 1.

Tabla 1. Abreviaturas y fórmulas básicas.

El trabajo experimental se ha realizado sobre un motor Fiat de la serie 138A.028 de $1498 \mathrm{~cm}^{3}$ de cilindrada. Se han tomado como datos de partida la carrera, el diámetro del cilindro, el número de cilindros y la curva característica de potencia. Estos datos han sido obtenidos del manual de taller del

\begin{tabular}{|c|c|c|c|}
\hline \multicolumn{4}{|c|}{ CUADRO DE ABREVIATURAS Y FORMULAS BÁSICAS.- } \\
\hline Abreviatura & Parámetro & Unidad & Fórmula \\
\hline $\mathrm{C}$ & Carrera & $(\mathrm{cm})$ & dato \\
\hline D & Diámetro & $(\mathrm{cm})$ & dato \\
\hline$\varepsilon$ & Relalción de compresión & $* * *$ & $\varepsilon=V_{1} / N 2$ \\
\hline Vc & Cilindrada unitaria & $(\mathrm{cm} 3)$ & $\mathrm{V} c=\pi \cdot \mathrm{D} 2 \cdot \mathrm{C} / 4$ \\
\hline$N^{\circ}$ & $\mathrm{N}^{\circ}$ de cilindros & $* * *$ & dato \\
\hline Vet & Cilindrada Total & $(\mathrm{cm} 3)$ & Vct $=$ Vc. $\mathrm{N}^{\bullet}$ cil. \\
\hline $\mathrm{N}$ & Regimen de rotación del motor & (1/min) & dato \\
\hline$\sqrt{ } 2$ & Volumen de la cámara de combustón & $(\mathrm{cm} 3)$ & medido \\
\hline V1 & Volumen total del cilindro & $(\mathrm{cm} 3)$ & $\begin{array}{c}\mathrm{V} 1=\varepsilon . \mathrm{V} 2 ; \text { o bien } \\
\mathrm{V} 1=\mathrm{V} \mathrm{c}+\mathrm{V} 2\end{array}$ \\
\hline P1 & Presión de admisión & $(\mathrm{kg} / \mathrm{cm} 2)$ & medido \\
\hline $\mathrm{n}$ & $\begin{array}{l}\text { Exponente politrópico en la } \\
\text { transformación de compresión y } \\
\text { expansión }\end{array}$ & $* * *$ & ver formula (3) \\
\hline P2 & Presión final de compresión & $(\mathrm{kg} / \mathrm{cm} 2)$ & medido \\
\hline
\end{tabular}
motor [2] y se muestran en la Tabla 2.

Tabla 1. Abreviaturas y fórmulas básicas. 
Tabla 2. Datos técnicos del motor y cálculos preliminares de los parámetros geométricos del motor $V_{1}$ y $\varepsilon$.

\begin{tabular}{|c|c|c|c|c|c|c|c|c|c|c|}
\hline \multirow{3}{*}{\multicolumn{2}{|c|}{$\begin{array}{l}\text { Aplicación del modelo del ciclo } \\
\text { previsto: ensayo preiminar. Valo } \\
\text { de P2 obtenido a } 2450 \text { RPM. }\end{array}$}} & \multicolumn{6}{|c|}{ DATOS TEECNICOS DEL MOTOR Y RPM DE ENSAYO } & \multicolumn{3}{|c|}{ CALCULO GEOUETRIA CILINDRO } \\
\hline & & 1 & 2 & 3 & 4 & 5 & 6 & 7 & 11 & 12 \\
\hline & & Cerrera & Diametro & $N$ de olindros & $\begin{array}{l}\text { Cindrada } \\
\text { uatura }\end{array}$ & \begin{tabular}{c|} 
porencis \\
efectiva a 2450 \\
npm.
\end{tabular} & $\begin{array}{c}\text { Rotaciones } \\
\text { conteip. a la } \\
\text { potencia } \\
\text { ealuaca }\end{array}$ & $\begin{array}{l}\text { Redacionde } \\
\text { compresilse }\end{array}$ & $\begin{array}{l}\text { Volumen total } \\
\text { del ilindro }\end{array}$ & $\begin{array}{l}\text { Volumee de } \\
\text { cimera de } \\
\text { combustetn }\end{array}$ \\
\hline & & c & 0 & $\mathrm{~N}^{*}$ & $\mathrm{ve}$ & $\mathrm{Pe}$ & $\mathrm{N}$ & $\varepsilon$ & $\mathrm{n}_{1}$ & $\mathrm{v} 2$ \\
\hline & & $(\mathrm{mm})$ & $(\mathrm{mn})$ & & $(\mathrm{om} 3)$ & (CV) & (ippan) & & {$[\mathrm{mm} 3]$} & {$[\mathrm{cm} 3]$} \\
\hline & & \multirow{2}{*}{$\begin{array}{l}\text { datomanal } \\
\text { fatriantet }\end{array}$} & \multirow{2}{*}{$\begin{array}{l}\text { deso macual } \\
\text { fatricants }\end{array}$} & \multirow{2}{*}{$\begin{array}{l}\text { drom manual } \\
\text { fibricante }\end{array}$} & calcuasio & \multirow{2}{*}{$\begin{array}{l}\text { dato manus } \\
\text { fatriante }\end{array}$} & \multirow{2}{*}{$\begin{array}{l}\text { datomanual } \\
\text { fibricarte }\end{array}$} & calalads & Celcuasio & \multirow{2}{*}{ netiso } \\
\hline Mecal Mocto & Clindro $\mathrm{N}^{*}$ & & & & $\psi_{c}=\pi .02 . c / 4$ & & & $\mathrm{~s}=\mathrm{V} 1 / \mathrm{V} 2$ & $v_{1}=v_{c}+v_{2}$ & \\
\hline \multirow{5}{*}{$\begin{array}{l}\text { Fint Acqunta 8s. } \\
1360 \mathrm{ccc}\end{array}$} & Qindro 1 & 63,9 & 86,4 & 1 & 374,64 & 9,27 & 2450 & 9,89 & 416,76 & 42,12 \\
\hline & Cindro 2 & 63,9 & 86,4 & 1 & 374,64 & 9,27 & 2450 & 10,11 & 415,76 & 41.12 \\
\hline & Qindro 3 & 63,9 & 86,4 & 1 & 374,64 & 9,27 & 2450 & 10,11 & 415,76 & 41,12 \\
\hline & Cindro 4 & 63,9 & 86,4 & 1 & 374,64 & 9,27 & 2450 & 10,11 & 415,76 & 41,12 \\
\hline & TOTALES & & & 4 & 1498,57 & 37,08 & & & & \\
\hline
\end{tabular}

3. PROCEDIMIENTO EXPERIMENTAL Y RESULTADOS OBTENIDOS.

3.1 Determinación de la máxima presión de compresión $\mathrm{P}_{2}$ y el coeficiente politrópico de la curva de compresión.

El valor de la presión final de compresión ha sido registrado con la ayuda de un manómetro especialmente preparado llamado compresómetro. Este manómetro es capaz de retener la lectura de la máxima presión que registra gracias a una válvula de retención instalada en su conexión de ingreso al tubo de Bourdón. E1 mismo fue instalado mediante un accesorio compuesto de una extensión que permite roscarlo en el orificio de la bujía.

Para este ensayo se instaló además, una válvula de venteo que permite encender el motor con el cilindro descomprimido para no someter al manómetro y sus accesorios a las altas temperaturas de compresión hasta el momento de realizar la medición.

A diferencia de los resultados presentados en [1] para esta etapa del proyecto CIPREV se realizaron mediciones con el motor en funcionamiento como sigue:

a) Se procedió al encendido del motor permitiéndole alcanzar la temperatura de régimen.

b) Se extrajo solo la bujía de encendido del cilindro 4 (en el que se realizó la medición de $\mathrm{P}_{2}$ ).

c) Se instaló el compresómetro en dicho cilindro.

d) Se encendió nuevamente el motor con el cilindro 4 descomprimido por la válvula de venteo.

e) Se aplicó carga al motor con el freno dinamométrico, hasta alcanzar el 100\% de carga a un régimen de 2450 rotaciones por minuto.

f) $\mathrm{Al} 100 \%$ de carga y a rpm constantes se procedió a cerrar la válvula de venteo y registrar en el cilindro 4 la máxima presión de compresión $\mathrm{P}_{2}$ en condiciones "cuasi reales" de funcionamiento y comprimiendo mezcla (es decir fluido de trabajo real). 
Tabla 3. Resultados experimentales del ensayo de compresión.

\begin{tabular}{|c|c|c|c|c|c|c|c|}
\hline \multicolumn{8}{|c|}{ 1.- PRESIÓN FINAL DE COMPRESIÓN (P2) } \\
\hline Codigo & \multicolumn{4}{|c|}{ EP 002} & Fecha & \multicolumn{2}{|c|}{$12 / 11 / 2014$} \\
\hline $\mathrm{N}^{\circ}$ de ensayo & \multicolumn{4}{|c|}{ EP 002-005/14 } & Duración (n) & \multicolumn{2}{|c|}{$2.30 \mathrm{hs}$} \\
\hline Laboratorio & \multicolumn{4}{|c|}{ Termofluidos.- F.I.- UdeMM.- } & & & \\
\hline \multicolumn{8}{|c|}{ 1.2-RESULTADOS EXPERIMENTALES: } \\
\hline $\begin{array}{c}\text { VALOR EXP. } \\
\text { COMPRESOME } \\
\text { TRO }\end{array}$ & \multicolumn{4}{|c|}{ VALORES EXPERIMENTALES FRENO DINAMOMÉTRICO } & \multicolumn{3}{|c|}{$\begin{array}{l}\text { VALORES EXP. ESTACÓN } \\
\text { METEOROLOGICA }\end{array}$} \\
\hline $\begin{array}{c}\text { Presión final de } \\
\text { compresión del } \\
\text { Cilindro } \mathrm{N}^{4} 4\end{array}$ & Fuerza & RPM & Estado carga & P adm. & $\begin{array}{l}\text { Humedad } \\
\text { relativa }\end{array}$ & $\begin{array}{c}\text { Temperatura } \\
\text { ambiente }\end{array}$ & $\begin{array}{c}\text { Presión } \\
\text { atmostéria }\end{array}$ \\
\hline$P_{2 C 4}$ & $F$ & & & P1 & $\mathrm{Hr}$ & Tamb & Patm \\
\hline$\left[\mathrm{kg} / \mathrm{cm}^{2}\right]$ & $(\mathrm{kg})$ & $(1 / \mathrm{min})$ & $(\%)$ & $(\mathrm{cmHg})$ & (\%) & ('C) & $(\mathrm{hPa})$ \\
\hline Medido & Medido & Medido & Medido & Medido & Medido & Medido & Medido \\
\hline 13,75 & 12,3 & 2450,0 & 100,00 & 4 & 45 & 24,5 & 1013 \\
\hline
\end{tabular}

\subsection{Determinación del consu-} mo específico y gases de combustión.
Tabla 4. Medición de los productos de combustión durante el ensayo de potencia al freno

Estas determinaciones fueron realizadas mediante un ensayo de potencia al freno convencional con el motor funcionando normalmente al 100\% de carga y un número de rotaciones similar al del ensayo de determinación de $\mathrm{P}_{2} \mathrm{y}$ del coeficiente politrópico para que sus resultados pudieran ser comparables. Durante el mismo se sometió al motor a un régimen de $2117 \mathrm{rpm}$ durante un tiempo de 9,5 minutos. Observándose una potencia efectiva al freno de $39,88 \mathrm{CV}$, un par motor de 13,49 kgm y resultando el consumo específico $0,1628 \mathrm{~kg} / \mathrm{CV} . \mathrm{h}$.

De este ensayo se pudo obtener la cantidad de combustible que participa de cada ciclo de trabajo del motor, cuyo valor medio fue de 1,02226 x10-4kg de combustible/ ciclo.

\begin{tabular}{|c|c|c|c|c|}
\hline \multicolumn{5}{|c|}{ 3.- MEDICIÓN DE GASES DE COMBUSTIÓN } \\
\hline Codigo & EP 003 & $\mathrm{~N}^{5}$ ensayo & EP $003-003 / 15$ & $\begin{array}{c}\text { Fecha: } \\
11 / 08 / 2015\end{array}$ \\
\hline Duracion & $9,5 \mathrm{~min}$ & Laboratorio & \multicolumn{2}{|c|}{$\begin{array}{c}\text { Termofluidos.- F.I.- } \\
\text { UdeMM.- }\end{array}$} \\
\hline \multicolumn{5}{|c|}{ 3.1- CONDICIONES DE ENSAYO.- } \\
\hline \multicolumn{2}{|c|}{ Vel. De rotación (RPM) } & 2116,87 & \multirow{2}{*}{\multicolumn{2}{|c|}{$\begin{array}{c}\text { Caract. Teorica del } \\
\text { combustible }\end{array}$}} \\
\hline \multicolumn{2}{|c|}{ Fuerza $(\mathrm{kg})$} & 18.84 & & \\
\hline \multicolumn{2}{|c|}{ Potencia (CV) } & 39.88 & \multicolumn{2}{|c|}{$\frac{\text { combustible }}{\mathrm{C} 8 \mathrm{H} 18}$} \\
\hline \multicolumn{2}{|c|}{ Par Motor (kgm) } & 13,49 & \multicolumn{2}{|c|}{ Masa molecular } \\
\hline \multicolumn{2}{|c|}{ Cons. Especifico (kg/CV.h) } & 0,1628 & \multicolumn{2}{|c|}{$114 \mathrm{~kg} / \mathrm{kmol}$} \\
\hline \multicolumn{2}{|c|}{ Cons. p/ciclo de trabajo $(\mathrm{kg})$} & $1.02226 \times 10^{2}$ & \multicolumn{2}{|c|}{ Aire estequeom étrico } \\
\hline \multicolumn{2}{|c|}{ Pc teórico del comb. (kcal//kg) } & 10.526 .30 & \multicolumn{2}{|c|}{$15.25 \mathrm{~kg} / \mathrm{kg} \mathrm{comb}$} \\
\hline \multicolumn{5}{|c|}{ 3.2-RESULTADOS EXPERIMENTALES.- } \\
\hline \multicolumn{4}{|c|}{ Variable medida } & \multirow{2}{*}{$\begin{array}{l}\text { Valor } \\
\text { medido }\end{array}$} \\
\hline orden & Magnitud & \begin{tabular}{|c|}
$\begin{array}{c}\text { Abreviatura/ } \\
\text { Simbolo }\end{array}$ \\
\end{tabular} & Unidad & \\
\hline 1 & Oxigeno libre & $\mathrm{O} 2$ & $\%$ & 1,0 \\
\hline 2 & Monowdo Carbono & $\mathrm{CO}$ & ppm & 4297 \\
\hline 3 & Exceso de aire & $\lambda$ & (................ & 1,05 \\
\hline 4 & Diónido de Carbono & $\mathrm{CO} 2$ & $\%$ & 14,76 \\
\hline 5 & Perdda por humos & $\mathrm{pA}$ & $\%$ & 14,7 \\
\hline 6 & Tiro & 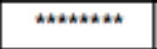 & $\mathrm{hPa}$ & 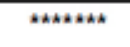 \\
\hline 7 & Temp. Ambiente & TA & ${ }^{\circ} \mathrm{C}$ & 21,1 \\
\hline 8 & $\begin{array}{l}\text { Temp oe los productos } \\
\text { de Combustion }\end{array}$ & $\mathrm{TH}$ & ${ }^{\circ} \mathrm{C}$ & 386,4 \\
\hline 9 & $\begin{array}{l}\text { Rendimiento de la } \\
\text { cambussón }\end{array}$ & Ren & $\%$ & 85,2 \\
\hline
\end{tabular}

Al mismo tiempo, mientras se desarrollaba el ensayo de potencia al freno, utilizando un analizador portátil de gases de combustión Testo se realizaron mediciones

de los productos de combustión para la situación real de ensayo. Los resultados experimentales se muestran en la Tabla 5. 


\section{RESULTADOS.}

4.1 Aplicación y resultados del modelo del ciclo indicado previsto.

A continuación se muestra como se opera con el valor obtenido experimentalmente para la máxima presión de compresión real
$\mathrm{P}_{2}$, obteniendo el coeficiente politrópico que caracteriza al fluido real y al sistema particular. En la tabla 5 se puede observar como a partir de los datos iniciales, se han obtenidos los valores correspondientes de $\mathrm{T}_{2}$, el trabajo de compresión $\mathrm{L}_{\mathrm{c}}$ y la presión media ficticia de la carrera de compresión.

Tabla 5. Resultados de aplicación del método del ciclo previsto a la medición realizada sobre el cilindro $\mathrm{n}^{\circ} 4$, al 100\% de carga y $2450 \mathrm{rpm}$.

\begin{tabular}{|c|c|c|c|c|c|c|c|c|}
\hline \multirow{4}{*}{\multicolumn{2}{|c|}{$\begin{array}{l}\text { Aplicación del modelo del } \\
\text { ciclo previsto: ensayo } \\
\text { preliminar. Valor de P2 } \\
\text { obtenido a } 2450 \text { RPM.- } \\
\text { Determinación del } \\
\text { exponente n }\end{array}$}} & \multicolumn{7}{|c|}{ CALCULO DEL CICLO PREVISTO PARA EL CILINDRO N4.- SOLO FASE DE COMPRESIÓN.- } \\
\hline & & 13 & 14 & 15 & 16 & 17 & 20 & 21 \\
\hline & & $\begin{array}{c}\text { Presión de } \\
\text { admisión/ inicio } \\
\text { compresión }\end{array}$ & $\begin{array}{l}\text { Temperatura } \\
\text { inicio } \\
\text { compresión }\end{array}$ & $\begin{array}{l}\text { Exponente } \\
\text { politrópico de } \\
\text { compresion }\end{array}$ & $\begin{array}{c}\text { Presión final de } \\
\text { compresión }\end{array}$ & $\begin{array}{l}\text { Temperatura } \\
\text { final de } \\
\text { compresión }\end{array}$ & $\begin{array}{l}\text { Trabajo de } \\
\text { compresión }\end{array}$ & $\begin{array}{l}\text { Presión media } \\
\text { proceso } \\
\text { compresión }\end{array}$ \\
\hline & & P1 & $\mathrm{T1}$ & $n$ & P2 & $\mathrm{T} 2$ & Le & $\mathrm{pm}$ \\
\hline & & $(\mathrm{kg} / \mathrm{cm} 2)$ & $\left({ }^{\circ} \mathrm{C}\right)$ & & $(\mathrm{kg} / \mathrm{cm} 2)$ & $\left({ }^{\circ} \mathrm{C}\right)$ & $(\mathrm{kgm})$ & $(\mathrm{kg} / \mathrm{cm} 2)$ \\
\hline & & \multirow{2}{*}{ medido } & \multirow{2}{*}{$\begin{array}{l}\text { medido c/la } \\
\text { cámara } \\
\text { termografica }\end{array}$} & calculado & \multirow{2}{*}{ medido } & calculado & calculado & Calculado \\
\hline Marca/ Modelo & Cilindro $\mathrm{N}^{*}$ & & & formula (3) & & $T 2=T_{1} \cdot E^{\wedge}(n-1)$ & formula (9) & $\mathrm{pmi}=\mathrm{L} / \mathrm{Nc}$ \\
\hline \multirow{5}{*}{$\begin{array}{c}\text { Fiat Regatta } 85 \\
1500 \mathrm{cc} .\end{array}$} & Cilindro 1 & 0,95 & & \#INUMI & & HINUMI & HIREF! & HIREF! \\
\hline & Cilindro 2 & 0,95 & & \#INUM! & & HiNUM! & HIREF! & $\pi \mid$ REF! \\
\hline & Cilindro 3 & 0,95 & & \#iNUM! & & \#INUM! & \#iREF! & \#iREF! \\
\hline & Cilindro 4 & 0,95 & 72 & 1,185 & 14,75 & 256,86 & 11,41 & 3,05 \\
\hline & TOTALES & & & & & & & \\
\hline
\end{tabular}

La presión de admisión $\mathrm{P}_{1}$ fue medida durante el ensayo con la ayuda de un vacuómetro conectado a la admisión. La temperatura $T_{1}$ fue obtenida mediante la utilización de una cámara termográfica Testo, en el momento previo a la determinación de la máxima $\mathrm{P}_{2}$, fotografiando el venteo del dispositivo de descompresión del cilindro 4 .

\subsection{Cálculo de las características termo-} dinámicas del fluido de trabajo y del sistema paritcular.

Siendo $\alpha$ la relación entre la masa de aire $\mathrm{m}_{\mathrm{a}}$ y la masa de combustible $\mathrm{m}_{\mathrm{c}}$ conocida. $\mathrm{Y}$ tomando el exceso de aire medido por el analizador de gases de combustión, es po- sible determinar la masa total de mezcla aplicando (14).

Resultando para este caso particular, donde $\alpha=15,25$ y $\lambda=1,05$, que la masa total de fluido de trabajo $\mathrm{m}_{\mathrm{t}}=1,739119 \mathrm{x}$ $10^{-3} \mathrm{~kg}$. Aplicando (11) será posible obtener para cualquier punto de la politrópica de compresión el valor de la constantr $\mathrm{R}_{\mathrm{g} 1}$ que caracteriza al sistema particular. Aplicándola al inicio y al fin de compresión es posible plantear:

$$
R_{g 1}=\frac{p_{1} \cdot V_{1}}{m_{t} \cdot T_{1}} \text { y } \quad R_{g 1}=\frac{p_{2} \cdot V_{2}}{m_{t} \cdot T_{2}}
$$


Tabla 6. Cálculo de la constante $\mathrm{R}_{\mathrm{g} 1}$ para el inicio y final de compresión del sistema particular con fluido real.

\begin{tabular}{|c|c|c|c|c|c|c|c|c|}
\hline \multicolumn{9}{|c|}{ CALCULO DE LA CONSTANTE PARTICULAR DEL SISTEMA Rg1 } \\
\hline & \multicolumn{4}{|c|}{$\begin{array}{l}\text { VALORES CORRESPONDIENTES AL INICIO DE } \\
\text { COPRESIÓN }\end{array}$} & \multicolumn{4}{|c|}{$\begin{array}{c}\text { VALORES CORRESPONDIENTES AL FINAL DE } \\
\text { COMPRESIÓN }\end{array}$} \\
\hline 22 & 23 & 24 & 25 & 26 & 27 & 28 & 29 & 30 \\
\hline $\begin{array}{c}\text { Masa total de } \\
\text { Mezcla }\end{array}$ & $\begin{array}{c}\text { Presion } \\
\text { inicio } \\
\text { compresion }\end{array}$ & $\begin{array}{l}\text { Volumen total } \\
\text { cilindro }\end{array}$ & $\begin{array}{c}\text { Temperatura } \\
\text { inicio } \\
\text { compresion }\end{array}$ & $\begin{array}{c}\text { Constante } \\
\text { particualr del } \\
\text { sistema } \\
\text { calculada en } \\
1\end{array}$ & $\begin{array}{l}\text { Presion final } \\
\text { de } \\
\text { compresión }\end{array}$ & $\begin{array}{l}\text { Volumen } \\
\text { cámara de } \\
\text { combustión }\end{array}$ & $\begin{array}{c}\text { Temperatura } \\
\text { final de } \\
\text { compresion }\end{array}$ & $\begin{array}{l}\text { Constante } \\
\text { particualr del } \\
\text { sistema } \\
\text { calculada en } \\
2\end{array}$ \\
\hline $\mathrm{mt}$ & P1 & V1 & $\mathrm{T} 1$ & Rg1-1 & $\mathrm{P} 2$ & V2 & $\mathrm{T} 2$ & $\mathrm{Rg} 1-2$ \\
\hline$(\mathrm{kg})$ & $(\mathrm{kg} / \mathrm{m} 2)$ & $(\mathrm{m} 3)$ & $(\mathrm{K})$ & $(\mathrm{kgm} / \mathrm{kg} . \mathrm{K})$ & $(\mathrm{kg} / \mathrm{m} 2)$ & $(\mathrm{m} 3)$ & $(\mathrm{K})$ & $(\mathrm{kgm} / \mathrm{kg}, \mathrm{K})$ \\
\hline calculado (14) & mecido & calculado & medido & $\begin{array}{c}\text { calculado } \\
\text { (11) }\end{array}$ & medido & mecilo & calculado & $\begin{array}{c}\text { calculado } \\
\text { (11) }\end{array}$ \\
\hline $1,74 E-03$ & 9500 & $4,16 \mathrm{E}-04$ & 345,15 & 6,5800 & 147500 & $4,11 \mathrm{E}-05$ & 530,01 & 6,5801 \\
\hline
\end{tabular}

Aplicando (10) es posible determinar el calor específico a volumen constante del fluido de trabajo:

$$
c_{v}=\frac{L_{c}}{\left(T_{2}-T_{1}\right) \cdot m_{t}}
$$

$\mathrm{E} 1 \mathrm{c}_{\mathrm{v}}=35,49 \mathrm{kgm} / \mathrm{kg} . \mathrm{K}$, o bien aplicando el equivalente calórico del trabajo $\mathrm{A}=$ $1 / 427(\mathrm{kcal} / \mathrm{kgm}), \mathrm{c}_{\mathrm{v}}=0,083114 \mathrm{kcal} / \mathrm{kg} . \mathrm{K}$. Y aplicando la relación de Mayer:

$$
R_{g 1}=c_{p}-c_{v}
$$

De donde el calor específico a presión constante para el fluido de trabajo real en el sistema particular resulta $c_{v}=0,09852383$ $\mathrm{kcal} / \mathrm{kg} . \mathrm{K}$.

\section{CONCLUSIONES.}

Como se ha podido apreciar el método para la obtención del coeficiente politrópico aplicado al ciclo previsto de un motor de encendido a chispa combinado con un análisis de gases de combustión durante el ensayo de potencia al freno, ha permitido determinar características termodinámicas fundamentales del fluido real de trabajo. Este es el caso de la constante particular del sistema $\mathrm{R}_{\mathrm{g} 1}$, el calor específico a volumen constante $c_{v}$ y el calor específico a presión constante $c_{p}$.

Como estos datos caracterizan al sistema particular, es decir al sistema conformado por el cilindro del motor y el fluido en las condiciones de trabajo, pueden tomarse como un parámetro de diseño para el cálculo de ciclos comparables con el estudiado.

La técnica descripta en 3.1 muestra la importancia de no circunscribir a los conocidos ensayos tradicionales a los motores de combustión interna. Existen una serie de ensayos no convencionales que permiten inferir características importantes que hacen a la determinación de parámetros que pueden tomarse como de diseño, y sirven al proyectista para el cálculo del calor producido, el trabajo y la potencia entre otros. 


\section{REFERENCIAS}

1. Caputo DC, Berberi RO, Ferré NAE, Bruno JM, Calvo $\mathrm{BE}$, Aguirre RN: Determinación del exponente politrópico y su uso en el modelo del ciclo indicado previsto en motores a gasolina. IV CAIM 2014, Univ. Nacional del Nordeste, Resistencia, Chaco.

2. Fiat Regatta Manual de Taller. Sevell Argentina S.A.

3. Martinez de Vedia, H: Teoría de los Motores Térmicos
Conversión de la Energía. Alsina, Argentina (1997).

4. Barilá D, Bugna L, Vignolo F, Gomez R, Kolodka P, Ahrtz G: Obtención del diagrama indicado en motores de combustión interna sin necesidad de referencia angualr. I CAIM 2008, Univ. Nacional del Sur, Bahía Blanca, Buenos Aires.

5. Jóvaj, MS.: Motores de Automóvil, Mir, Moscú (1987). 